\title{
Killer-cell Immunoglobulin-like Receptor (KIR) gene profiles modify HIV disease course, not HIV acquisition in South African women
}

\author{
V. Naranbhai ${ }^{1,2,3^{*}}$ D, D. de Assis Rosa ${ }^{4,5}$, L. Werner ${ }^{1}$, R. Moodley $^{3}$, H. Hong ${ }^{13}$, A. Kharsany ${ }^{1}$, K. Mlisana', S. Sibeko ${ }^{1}$, \\ N. Garrett ${ }^{1}$, D. Chopera ${ }^{6}$, W. H. Carr ${ }^{3,7,8}$, Q. Abdool Karim ${ }^{1,9}$, A. V. S. Hill ${ }^{2}$, S. S. Abdool Karim ${ }^{1,9}$, M. Altfeld ${ }^{8,10}$, \\ C. M. Gray ${ }^{4,6^{*}}$ and T. Ndung ' $\mathbf{u}^{3,8,11,12^{*}}$
}

\begin{abstract}
Background: Killer-cell Immunoglobulin-like Receptors(KIR) interact with Human Leukocyte Antigen(HLA) to modify natural killer- and T-cell function. KIR are implicated in HIV acquisition by small studies that have not been widely replicated. A role for KIR in HIV disease progression is more widely replicated and supported by functional studies.

Methods: To assess the role of KIR and KIR ligands in HIV acquisition and disease course, we studied at-risk women in South Africa between 2004-2010. Logistic regression was used for nested case-control analysis of 154 women who acquired vs. 155 who did not acquire HIV, despite high exposure. Linear mixed-effects models were used for cohort analysis of 139 women followed prospectively for a median of 54 months (IQR 31-69) until 2014.

Results: Neither KIR repertoires nor HLA alleles were associated with HIV acquisition. However, KIR haplotype BB was associated with lower viral loads $(-0.44 \log 10$ copies $/ \mathrm{ml} ; \mathrm{SE}=0.18 ; \mathrm{p}=0.03)$ and higher CD4+ T-cell counts $(+80$ cells/ $\mu l ; S E=42 ; p=0.04$ ). This was largely explained by the protective effect of KIR2DL2/KIR2DS2 on the B haplotype and reciprocal detrimental effect of KIR2DL3 on the A haplotype.
\end{abstract}

Conclusions: Although neither KIR nor HLA appear to have a role in HIV acquisition, our data are consistent with involvement of KIR2DL2 in HIV control. Additional studies to replicate these findings are indicated.

Keywords: KIR, HLA, HIV, Acquisition, Viral control, Disease progression

\section{Background}

An array of host genetic factors have been reported to alter HIV acquisition or markers of HIV disease progression $[1,2]$. Identifying and understanding these genetic correlates may accelerate preventive and therapeutic efforts.

The most widely replicated correlates of HIV acquisition include homozygosity in the 32-base pair deletion in CCR5 that reduces acquisition risk. Although, in comparison, greater effort has been focused on identifying correlates of HIV disease progression there are similarly only a

\footnotetext{
*Correspondence: vivekn@well.ox.ac.uk; clive.gray@uct.ac.za; ndungu@ukzn. ac.za

${ }^{1}$ Centre for the AIDS Programme of Research in South Africa (CAPRISA), University of KwaZulu-Natal, Durban, South Africa

${ }^{4}$ National Institute of Communicable Diseases, Sandringham, South Africa

${ }^{3}$ HIV Pathogenesis Programme, University of KwaZulu-Natal, Durban, South Africa

Full list of author information is available at the end of the article
}

handful of widely replicated findings. Natural Killer (NK) cell and CD8+ T-cell responses stand out as being reproducibly implicated in HIV control [3]. Concordantly, variation in the Human Leukocyte Antigen (HLA) and Killer-cell Immunoglobulin-like Receptor (KIR) loci, the two most polymorphic regions of the human genome that encode receptors involved in NK and CD8+ T-cell function, is associated with rates of HIV disease progression across several studies [2]. Genome-wide association studies identify $H L A-B * 57$ alleles, and variants that affect HLA-C expression [4-6] as important modifiers of HIV viraemia, the former of which had been identified in many earlier candidate gene studies.

Natural Killer cells are amongst the earliest responders to viral infection and mediate protective responses by secreting pro-inflammatory cytokines and by direct cytolysis of infected cells. Their function is governed, at least in part, 
by the combinatorial array of inhibitory and activating receptors including the KIR, Leukocyte immunoglobulin-like receptors (LILR), the C-type lectin receptors-NKG2A-F, and the natural cytotoxic receptors (NCRs) -NKp30, NKp44 and NKp46. The dominant regulators of NK cell recognition of virus-infected cells are thought to be KIR, because these are the natural receptors for HLA class I [7]. Alteration in HLA expression and presentation of pathogen-derived or self-peptides is a common feature of many viral infections [8]. Diversity in KIR gene content, polymorphism and structural variation within the 14 KIR genes, and variation in expression confer additional variation in the ability of NK cells to identify and respond to virus-infected cells. KIRs show partial specificity in their recognition of HLA ligands: KIR3DL1 and KIR3DS1 recognise HLA-A and HLA-B molecules with the Bw4 epitope, KIR2DL1 recognises HLA-C molecules of the C2 group exclusively, KIR2DL3 recognises HLA-C of the $\mathrm{C} 1$ group exclusively and KIR2DL2 recognizes HLA-C of both C1 and C2 groups. The KIR genes segregate in two groups of haplotypes. Group A haplotypes consist of nine genes that encode predominantly inhibitory receptors, whereas group B haplotypes represent a more diverse collection of haplotypes based on gene content and contain more activating KIRs compared with haplotype A.

Several combinations of KIR and HLA have been associated with protection from HIV acquisition [9-13], though these findings are based on small sample sizes and none have been replicated in the literature. In contrast, a large study reported in 2002, found that KIR3DS1 together with HLA Bw4 alleles encoding Isoleucine at position 80 (80I) are associated with slower disease progression [5]. Several additional lines of evidence from subsequent observational and functional studies support a role for KIR in HIV control. NK cells are expanded in primary HIV infection [14], the expansion is modified by specific KIR and ligand repertoires [15], the degree of HLA C ligand expression associates with protection $[4,16]$ and viruses sequenced from individuals with specific KIR show evidence of 'escape' mutation at sites that appear to alter recognition by KIR [17]. As in infection with EBV [18], CMV [19], HCV or HTLV-1 [20], KIR have also been shown to modulate Tcell responses to HIV [21].

We studied the role of HLA and KIR on risk of HIV acquisition and course of HIV viraemia and CD4+ T-cell counts through the first 5-10 years of infection in South African women infected with HIV-1 clade C in a nested case-control and prospective cohort study respectively. In prior studies in this cohort we have reported that a) innate immune cell activation is associated with enhanced HIV acquisition [22]; b) HIV-directed NK cells secreting IFN-y are associated with reduced HIV acquisition risk [23]; c) HIV acquisition results in profound alteration in NK cell function [24] and d) the rate of HIV disease progression is more rapid than similar cohorts elsewhere $[25,26]$. This current study strongly implicates KIR2DL2, belonging to the group B haplotype with HIV control.

\section{Methods \\ Study design and cohort accrual}

Between 2004 and 2010, we prospectively enrolled and followed-up women at risk for HIV acquisition in studies [27-29] conducted at two urban and one rural site in KwaZulu-Natal, South Africa (Fig. 1) [26]. We performed two analyses of KIR/HLA genetic profiles: a nested casecontrol analysis of HIV acquisition and a prospective cohort analysis of involvement in HIV disease course. For the nested case-control analysis we compared 154 women who acquired HIV to 155 who did not and for the cohort analysis, studied 139 women with viral load and CD4+ Tcell count measures.

Women at risk for HIV were extensively counselled on HIV risk reduction and provided with condoms in line with the highest standard of counselling at the time [27-29].

The median age at enrolment was 21 years (IQR 20-26 years). As previously reported [30], women who acquired HIV were younger than those who remained HIV negative (median age 23, IQR 20-26 vs. median age 33, IQR 25-40). All except one woman in this study self-identified as blackAfrican of Zulu ethnicity.

During follow-up in the AI study, clinical care was offered as per contemporary South African treatment guidelines including combination antiretroviral therapy when clinically indicated or if the CD4+ T-cell count declined to below $200 \mathrm{cells} / \mu \mathrm{l}$ or $350 \mathrm{cells} / \mu \mathrm{l}$ (updated according to evolving treatment guidelines). Participants were seen according to the following schedule: weekly to fortnightly for the first 3 months post HIV acquisition, monthly from months 3-12 and quarterly thereafter. At each visit women received comprehensive prevention counselling, clinical examination, viral load and CD4+ T-cell count measurements and additional clinically indicated investigations performed at an accredited laboratory (ISO15189) using previously described methods [28, 29, 31]. The last possible date of censorship in those who did not reach a progression endpoint was 1 August 2014. Women were censored at time of combination antiretroviral therapy (cART) initiation, death, loss to follow-up or withdrawal from the study.

\section{Ethics statement}

Participants gave their written and informed consent to participation in each study according to protocols approved by the Biomedical Research Ethics Committee of the University of KwaZulu-Natal (Ref 050/051, E013/04, E111/06), and relevant collaborating centres (University of Cape Town 025/2004, University of Witwatersrand M040202). 


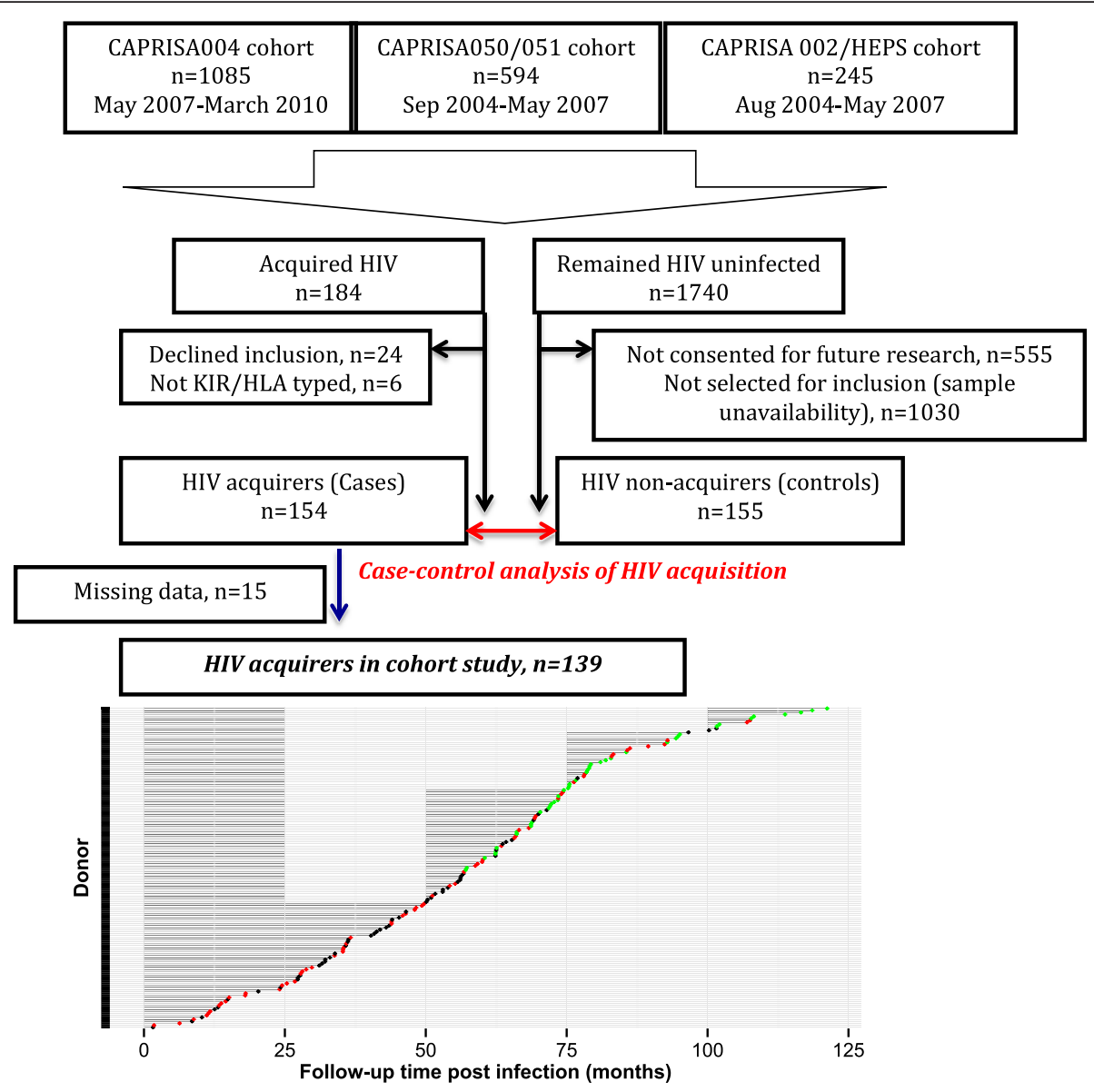

Fig. 1 Cohort accrual diagram and study design. The figure shows the three parent studies [27-29] in which HIV negative donors were enrolled, counselled on HIV risk reduction and followed-up prospectively and how the nested case-control subset was accrued. For the case control analysis of HIV acquisition, 154 women who acquired HIV were compared with 155 women who remained HIV negative through follow-up. For cohort analyses of HIV course, 154 women who acquired HIV and were followed up for up to 120 months were studied. Follow-up time and disposition as at 1 August 2014 (date of censorship), is shown in the lower panel for each donor where dot color denotes disposition (green: remains in follow-up, red: exited study as commenced cART or death, black:lost to follow-up)

The protocol under which analyses in this study are conducted was independently approved by the Biomedical Research Ethics Committee of the University of KwaZuluNatal (BE073/10).

\section{KIR and HLA typing}

Killer-cell immunoglobulin-like receptor genotyping was conducted in samples from 154 HIV-positive women and 155 HIV-negative women by sequence-specific oligonucleotide primer PCR or qPCR according to previously described methods [5, 32]. The definitions used to assign KIR haplotypes based on gene content were based on previous reports [7] and are described in detail in Additional file 1. Individuals having only and all genes of following group were denoted as having AA: KIR3DL3, KIR2DL3, KIR2DL1, KIR2DP1, KIR3DP1, KIR2DL4, KIR3DL1, KIR2 DS4, KIR3DL2. Individuals lacking any of the following were denoted as BB: KIR2DL1, KIR2DL3, KIR3DL1, KIR2
DS4. Individuals having all A haplotype genes and any 1 of the following genes were presumed to be AB: KIR 2DL2, KIR2DL5, KIR2DS1, KIRDS2, KIR2DS3, KIR 2DS5, KIR3DS1. Four-digit HLA typing was performed as previously described. Further information on classification of HLA alleles into Bw4 groups and $\mathrm{C} 1 / \mathrm{C} 2$ groups is given in Additional file 1.

\section{Outcome definition}

Methods of HIV diagnosis and estimation of the date of acquisition have been previously reported [27, 29]. Plasma HIV viral load was assessed by Roche Ampliprep/Amplicor or Roche Taqman (Roche Diagnostics, New Jersey, USA). CD4+ T-cell count enumeration in whole blood was performed by the TruCOUNT method on a FACSCalibur instrument (BD Biosciences, San Jose, USA). The median number of viral load and CD4+ T-cell count measures contributed per participant was 29 (IQR 22-34). 


\section{Statistical methods}

Statistical analyses were performed according to an $a$ priori statistical analysis plan (Additional file 1) with minor amendments made to update KIR haplotype definitions. For comparisons between women who acquired HIV and those who did not, logistic regression models were employed.

To accommodate potential inter- and intra-patient sources of variation we used linear mixed-effects models to study potential correlates of HIV viral load and CD4+ T-cell count decline because of their flexibility in allowing for possible heterogeneity in population, and potential imbalance in the longitudinal data. Graphical plotting of individual measures was performed using $\mathrm{R}$ software, and locally weighted scatterplot smoothing (LOWESS) was employed to derive aggregate curves. Analyses were performed in R using the following packages 'Ime4,' 'survival', 'ggplot2', 'rms' and 'LDheatmap'. P-values presented are nominal (unadjusted) p-values unless otherwise stated.

\section{Results}

\section{Cohort accrual}

Between May 2004 and March 2010, 1924 HIV negative women at risk for HIV acquisition were enrolled in one of three cohort studies in KwaZulu-Natal, South Africa and followed up to identify incident infection (Fig. 1). During a median of 16 months of active follow-up (IQR 7.823.7 months) with monthly or quarterly screening for HIV, 184 women acquired HIV (HIV incidence rate: 7.63 per 100 PY; 95 \% CI 6.59-8.78). Of these, 160 women (of whom 154 were KIR-genotyped) consented to inclusion in continued follow-up and were enrolled at a median of 42 (IQR 28-62) days post infection into the CAPRISA AI study. As at August 2014, the median follow-up was 54 (IQR 31-69) months and at this point 8 were lost to follow up, 8 had died, 1 withdrew from the study, 101 commenced cART at a median of 50 (IQR 32-65) months after infection and 42 remain in follow-up. For cohort analyses we excluded 15 individuals due to missing data, leaving 139 individuals with follow up data. The cumulative follow-up time was 629.3 woman-years.

\section{Neither KIR nor HLA profiles are associated with HIV acquisition}

To test whether KIR-HLA profiles are associated with risk of HIV acquisition we compared KIR gene content, and HLA alleles among 154 women (cases) who acquired HIV to 155 women (controls) who did not acquire infection during follow-up. With this sample size we had $80 \%$ power to detect predictors with odds ratio $<0.63$ or $>1.6$ at $\alpha=5 \%$ given the cohort HIV incidence of $\sim 7 \%$. The frequency of KIR genes, HLA alleles or groups, and KIRHLA ligand pairs was not associated with HIV acquisition in logistic regression models (Table 1).
Presence of the KIR haplotype BB is associated with lower viral loads during HIV infection and higher CD4+ T-cell counts

To test whether KIR or HLA are associated with differences in the course of disease we used a linear mixed model approach to model post-infection viral loads accounting for differences in times of viral load or CD4+ Tcell count measurement and inter-participant variation amongst 139 women.

The presence of KIR haplotype BB was significantly associated with lower viral loads (overall effect: $-0.44 \log _{10}$ copies $/ \mathrm{ml}, \mathrm{p}=0.03$, Table 2 and Fig. 2a). This observation was consistent regardless of the cohort from which these women were enrolled (Additional file 2: Figure S1). Concordantly, CD4+ T-cell counts were higher in individuals with $\mathrm{BB}$ compared with $\mathrm{AA} / \mathrm{AB}$ haplotypes (overall effect: +80 cells $/ \mu \mathrm{l}, \mathrm{p}=0.04$, Fig. $2 \mathrm{~b}$ ). Data beyond around 60 months of follow-up are sparse leading to overlapping confidence intervals thereafter. Crosssectional analysis of viral loads supports this observation (Additional file 3: Figure S2). Amongst 24 women for whom CD4 + T-cell counts prior to HIV acquisition were available, the counts did not differ according to haplotype $\left(\mathrm{n}_{\mathrm{AA}}=6, \mathrm{n}_{\mathrm{AB}}=14\right.$ and $\mathrm{n}_{\mathrm{BB}}=4 ; \operatorname{median}_{\mathrm{AA}}=914$, median $_{\mathrm{AB}}=981$ and median $_{\mathrm{BB}}=824$ cells $/ \mu \mathrm{l}$, KruskalWallis rank sum test $\chi^{2}=1.8, \mathrm{p}=0.4$ ) and the time of enrolment following HIV acquisition did not significantly differ between groups.

Classical HLA class I alleles grouped by presence/absence of Bw4/Bw6 epitopes or $\mathrm{C} 1 / \mathrm{C} 2$ groups were not, on their own, associated with course of viraemia but previously reported protective HLA alleles ( $B * 13: 02$, $B * 27, B * 57, B * 58: 01$ or $B * 81: 01)$ tended to be associated with lower viral loads (effect: $-0.27 \log _{10}$ copies $/ \mathrm{ml} \mathrm{p}=$ $0.10)$, and harmful alleles $(B * 18: 01, B * 35$ or $B * 58: 02)$ with higher viral loads (effect: $+0.25 \log _{10}$ copies $/ \mathrm{ml} \mathrm{p}=0.06$, Additional file 4: Figure S3). Inclusion of HLA alleles in the model did not attenuate association between KIR and course of HIV viraemia.

\section{Allelic state of KIR2DL2/KIR2DL3 may explain KIR genotype effects on HIV viral loads}

Next, we examined whether the presence of specific KIR on the B haplotype may explain the observation above. In contrast to previous reports, the absolute number of activating or inhibitory KIR-ligand pairs did not associate with HIV viraemia (data not shown). However, the presence of KIR2DL2 was associated with reduced HIV viral loads through the first 3-4 years of HIV and conversely, the presence of one or two copies of KIR2DL3 was significantly associated with elevated HIV viral loads $\left(+0.33\right.$ and $+0.46 \log _{10}$ copies $/ \mathrm{ml}$ respectively relative to KIR2DL2/KIR2DL2, $\mathrm{p}=0.02)$. 
Table 1 Results of logistic regression model of KIR/HLA and HIV acquisition

\begin{tabular}{|c|c|c|c|c|c|c|c|c|c|}
\hline & \multicolumn{3}{|c|}{ HIV- $(n=155)$} & \multicolumn{3}{|c|}{$\mathrm{HIV}+(\mathrm{n}=154)$} & \multirow[t]{2}{*}{$p^{a}$} & \multirow[t]{2}{*}{$O R^{a}$} & \multirow[t]{2}{*}{$95 \% \mathrm{Cl}^{\mathrm{a}}$} \\
\hline & - & + & $+\%$ & - & + & $+\%$ & & & \\
\hline \multicolumn{10}{|l|}{ KIR loci } \\
\hline KIR2DL1 & 4 & 151 & $97.4 \%$ & 0 & 154 & $100.0 \%$ & 0.98 & - & - \\
\hline KIR2DL2 & 45 & 110 & $71.0 \%$ & 46 & 107 & $69.9 \%$ & 0.89 & 1.04 & $0.63-1.71$ \\
\hline KIR2DL2/KIR2DL2 & & 39 & $25.5 \%$ & & 35 & $22.9 \%$ & & & \\
\hline KIR2DL2/KIR2DL3 & & 70 & $45.8 \%$ & & 72 & $47.1 \%$ & 0.63 & 1.15 & $0.43-2.05$ \\
\hline KIR2DL3/KIR2DL3 & & 44 & $28.8 \%$ & & 46 & $30.1 \%$ & 0.83 & 1.07 & $0.32-2.00$ \\
\hline KIR2DL3 & 40 & 114 & $74.0 \%$ & 35 & 118 & $77.1 \%$ & 0.61 & 1.15 & $0.68-1.94$ \\
\hline KIR2DL4 & 3 & 152 & $98.1 \%$ & 0 & 154 & $100.0 \%$ & 0.98 & - & - \\
\hline KIR2DL5 & 44 & 111 & $71.6 \%$ & 56 & 98 & $63.6 \%$ & 0.18 & 0.72 & $0.44-1.16$ \\
\hline KIR3DL1 & 0 & 155 & $100.0 \%$ & 0 & 154 & $100.0 \%$ & - & - & - \\
\hline KIR3DL1/KIR3DL1 & & 145 & $94.8 \%$ & & 141 & $92.2 \%$ & & & \\
\hline KIR3DL1/KIR3DS1 & & 8 & $5.2 \%$ & & 12 & $7.8 \%$ & 0.27 & 1.69 & $0.62-4.04$ \\
\hline KIR3DS1/KIR3DS1 & & 0 & $0.0 \%$ & & 0 & $0.0 \%$ & - & - & - \\
\hline KIR3DS1 & 145 & 8 & $5.2 \%$ & 141 & 12 & $7.8 \%$ & 0.27 & 1.69 & $0.67-4.45$ \\
\hline KIR3DL2 & 0 & 155 & $100.0 \%$ & 0 & 154 & $100.0 \%$ & - & - & - \\
\hline KIR3DL3 & 2 & 153 & $98.7 \%$ & 0 & 154 & $100.0 \%$ & 0.98 & - & - \\
\hline KIR2DS1 & 135 & 18 & $11.8 \%$ & 130 & 24 & $15.6 \%$ & 0.23 & 1.51 & $0.78-2.96$ \\
\hline KIR2DS2 & 63 & 92 & $59.4 \%$ & 60 & 94 & $61.0 \%$ & 0.56 & 1.15 & $0.72-1.83$ \\
\hline KIR2DS3 & 104 & 51 & $32.9 \%$ & 111 & 43 & $27.9 \%$ & 0.43 & 0.82 & $0.50-1.34$ \\
\hline KIR2DS3/KIR2DS3 & & 29 & $25.9 \%$ & & 24 & $24.2 \%$ & & & \\
\hline KIR2DS3/KIR2DS5 & & 22 & $19.6 \%$ & & 19 & $19.2 \%$ & 0.77 & 1.13 & $0.49-2.60$ \\
\hline KIR2DS5/KIR2DS5 & & 61 & $54.5 \%$ & & 56 & $56.6 \%$ & 0.77 & 1.11 & $0.57-2.14$ \\
\hline KIR2DS5 & 72 & 83 & $53.5 \%$ & 79 & 75 & $48.7 \%$ & 0.47 & 0.85 & $0.54-1.33$ \\
\hline $\mathrm{KIR} 2 \mathrm{DS} 4^{\mathrm{b}}$ & 2 & 153 & $98.7 \%$ & 1 & 153 & $99.4 \%$ & 0.63 & 1.80 & $0.17-39.03$ \\
\hline 197/. & & 23 & $20.2 \%$ & & 11 & $19.0 \%$ & & & \\
\hline 197/219 & & 31 & $27.2 \%$ & & 25 & $43.1 \%$ & 0.25 & 1.57 & $0.70-4.21$ \\
\hline.$/ 219$ & & 60 & $52.6 \%$ & & 22 & $37.9 \%$ & 0.55 & 1.56 & $0.32-1.87$ \\
\hline \multicolumn{10}{|l|}{ KIR Haplotype } \\
\hline $\mathrm{Bx}$ & 33 & 121 & $78.6 \%$ & 40 & 113 & $73.9 \%$ & 0.45 & 0.81 & $0.48-1.39$ \\
\hline AA & & 33 & $21.4 \%$ & & 40 & $26.1 \%$ & & & \\
\hline$A B$ & & 77 & $50.0 \%$ & & 78 & $51.0 \%$ & 0.65 & 0.88 & $0.50-1.55$ \\
\hline BB & & 44 & $28.6 \%$ & & 35 & $22.9 \%$ & 0.27 & 0.70 & $0.36-1.33$ \\
\hline \multicolumn{10}{|l|}{ HLA Groups } \\
\hline Protective B alleles & 122 & 33 & $21.3 \%$ & 128 & 26 & $16.9 \%$ & 0.33 & 0.75 & $0.42-1.33$ \\
\hline Harmful B alleles & 108 & 47 & $30.3 \%$ & 104 & 50 & $32.5 \%$ & 0.80 & 1.07 & $0.66-1.73$ \\
\hline Bw4 & 109 & 46 & $29.7 \%$ & 103 & 51 & $33.1 \%$ & 0.50 & 1.18 & $0.73-1.92$ \\
\hline Bw6 & 75 & 80 & $51.6 \%$ & 77 & 77 & $50.0 \%$ & 0.70 & 0.92 & $0.58-1.44$ \\
\hline $\mathrm{C} 1 / \mathrm{C} 1$ & & 21 & $13.5 \%$ & & 18 & $11.7 \%$ & & & \\
\hline $\mathrm{C} 1 / \mathrm{C} 2$ & & 68 & $43.9 \%$ & & 73 & $47.4 \%$ & 0.76 & 1.35 & $0.65-2.81$ \\
\hline $\mathrm{C} 2 / \mathrm{C} 2$ & & 66 & $42.6 \%$ & & 63 & $40.9 \%$ & 0.70 & 1.18 & $0.56-2.52$ \\
\hline \multicolumn{10}{|l|}{ KIR/HLA Ligand Pair } \\
\hline KIR2DL1_C2 & 33 & 122 & $78.7 \%$ & 25 & 129 & $83.8 \%$ & 0.22 & 1.44 & $0.81-2.59$ \\
\hline KIR2DS1_C2 & 140 & 15 & $9.7 \%$ & 132 & 22 & $14.3 \%$ & 0.15 & 1.68 & $0.84-3.46$ \\
\hline
\end{tabular}


Table 1 Results of logistic regression model of KIR/HLA and HIV acquisition (Continued)

\begin{tabular}{llllllllll}
\hline KIR2DL2_C1 & 90 & 65 & $41.9 \%$ & 92 & 62 & $40.3 \%$ & 0.77 & 0.93 & $0.59-1.47$ \\
KIR2DL3_C1 & 94 & 61 & $39.4 \%$ & 85 & 69 & $44.8 \%$ & 0.43 & 1.20 & $0.76-1.90$ \\
KIR3DL1_Bw4 & 109 & 46 & $29.7 \%$ & 103 & 51 & $33.1 \%$ & 0.50 & 1.18 & $0.73-1.92$ \\
KIR3DS1_Bw4 & 150 & 5 & $3.2 \%$ & 148 & 6 & $3.9 \%$ & 0.68 & 1.29 & $0.38-4.59$ \\
KIR2DS4_CW04 & 128 & 27 & $17.4 \%$ & 128 & 26 & $16.9 \%$ & 0.86 & 1.04 & $0.57-1.91$ \\
KIR3DL2_A3A11 & 134 & 21 & $13.5 \%$ & 133 & 21 & $13.6 \%$ & 0.98 & 1.01 & $0.52-1.95$ \\
\hline
\end{tabular}

Previous Presentations: A part of these data was presented at the International Immunogenetics and HLA Workshop 2012. Liverpool, UK, 28 May-3 June 2012 a(adjusted for tenofovir gel use)

${ }^{\mathrm{b}}$ For a subset (172/309, $56 \%$ KIR2DS4 alleles were genotyped where 197 denotes the common deletion variant of KIR2DS4 that has a 22 bp deletion in exon 5

Table 2 linear mixed-effects model results for association between KIR/HLA and HIV viraemia in 139 women with incident HIV infection followed up for up to 10 years post infection

\begin{tabular}{|c|c|c|c|c|}
\hline & $\begin{array}{l}\text { VL Intercept } \\
\left(\log _{10} / \mathrm{ml}\right)\end{array}$ & $\begin{array}{l}\text { Effect } \\
\left(\log _{10} / \mathrm{ml}\right)\end{array}$ & SE & $\begin{array}{l}\text { nominal } \\
\text { p-value }\end{array}$ \\
\hline \multicolumn{5}{|l|}{ Fixed effects } \\
\hline \multicolumn{5}{|l|}{ Haplotype } \\
\hline $\mathrm{AA}$ & 4.52 & Ref & 0.15 & 0.03 \\
\hline$A B$ & & -0.08 & 0.15 & \\
\hline $\mathrm{BB}$ & & -0.44 & 0.18 & \\
\hline \multicolumn{5}{|l|}{ KIR loci } \\
\hline KIR2DL2/KIR2DL2 & 4.08 & Ref & 0.15 & 0.02 \\
\hline KIR2DL2/KIR2DL3 & & 0.33 & 0.16 & \\
\hline KIR2DL3/KIR2DL3 & & 0.46 & 0.17 & \\
\hline KIR2DL5 & 4.43 & -0.13 & 0.13 & 0.32 \\
\hline KIR3DL1/KIR3DL1 & 4.33 & Ref & 0.10 & 0.84 \\
\hline KIR3DL1/KIR3DS1 & & -0.05 & 0.24 & \\
\hline KIR3DS1/KIR3DS1 & - & - & - & \\
\hline KIR2DS1 & 4.36 & -0.13 & 0.19 & 0.47 \\
\hline KIR2DS2 & 4.53 & -0.28 & 0.13 & 0.03 \\
\hline KIR2DS3/KIR2DS3 & 4.03 & Ref & 0.19 & 0.14 \\
\hline KIR2DS3/KIR2DS5 & & 0.01 & 0.25 & \\
\hline KIR2DS5/KIR2DS5 & & 0.33 & 0.20 & \\
\hline KIR2DS4 & 4.43 & -0.08 & 0.77 & 0.91 \\
\hline \multicolumn{5}{|l|}{ HLA groups } \\
\hline Protective HLA-B alleles & 4.38 & -0.27 & 0.17 & 0.10 \\
\hline Harmful HLA-B alleles & 4.26 & 0.25 & 0.13 & 0.06 \\
\hline Bw4 & 4.33 & 0.07 & 0.14 & 0.63 \\
\hline Bw6 & 4.39 & -0.08 & 0.13 & 0.52 \\
\hline Number of $\mathrm{C} 1$ epitopes & 4.40 & -0.08 & 0.09 & 0.40 \\
\hline Number of C2 epitopes & 4.25 & 0.07 & 0.09 & 0.43 \\
\hline Random Effects & & Variance & SD & \\
\hline Weeks postinfection & (Intercept) & 0.02 & 0.12 & \\
\hline X002PID & (Intercept) & 0.61 & 0.78 & \\
\hline Residual & & 0.31 & 0.55 & \\
\hline
\end{tabular}

The extensive linkage disequilibrium between KIR genes (Fig. 2c), can be leveraged to further understand this observation. Contrasting direction of effect between KIR2DL2 and KIR2DL3 and concordant protective effect of KIR2DS2 supports their involvement in HIV viral control because $K I R 2 D L 2$ and KIR2DL3 segregate as alleles of the same locus and KIR2DL2 and KIR2DS2 are in moderate linkage disequilibrium. Intriguingly, KIR2DL2/KIR2DL3 heterozygous individuals have viral loads that are similar to KIR2DL3/KIR2DL3 homozygous individuals suggesting that even one copy of KIR2DL3 is associated with elevated viraemia (Fig. 2d). A similar observation is noted when examining CD4+ T-cell counts (Fig. 2b). These data are consistent with the observation that BB haplotypes of KIR are associated with reduced viraemia as KIR2DL2 is a constituent of the B haplotype.

To assess whether KIR-ligand interactions may be involved we examined HIV viral loads according to gene content at the KIR2DL2/KIR2DL3 locus and their ligands: HLA alleles of the $\mathrm{C} 1 / \mathrm{C} 2$ groups (Additional file 5: Table S1). Linear mixed models testing for an interaction between KIR2DL2/KIR2DL3 locus gene content and the number of HLA C1/C2 ligands did not provide evidence of statistical interaction. Graphical examination of viral load measures and CD4+ T-cell counts support this interpretation (Additional file 6: Figure S4). Although larger studies may reveal nuanced effects, given the observations here the association between KIR2DL2/ KIR2DL3 and HIV viraemia does not appear to be discernably modified by HLA C1/C2 ligands.

\section{Discussion}

KIR and HLA haplotypes have been associated with HIV disease outome, but little is known about their role in HIV acquisition. Using a large cohort of prospectively enrolled women at risk for HIV, we did not observe evidence of association between KIR profiles and HIV-1 acquisition. This finding is consistent with recent studies that suggest that genetic variation does not explain a substantial proportion in liability to acquire HIV-1 [33, 34]. In contrast, we found that KIR genotype BB, which encodes KIR2DL2, was associated with lower HIV viraemia and higher CD4+ 

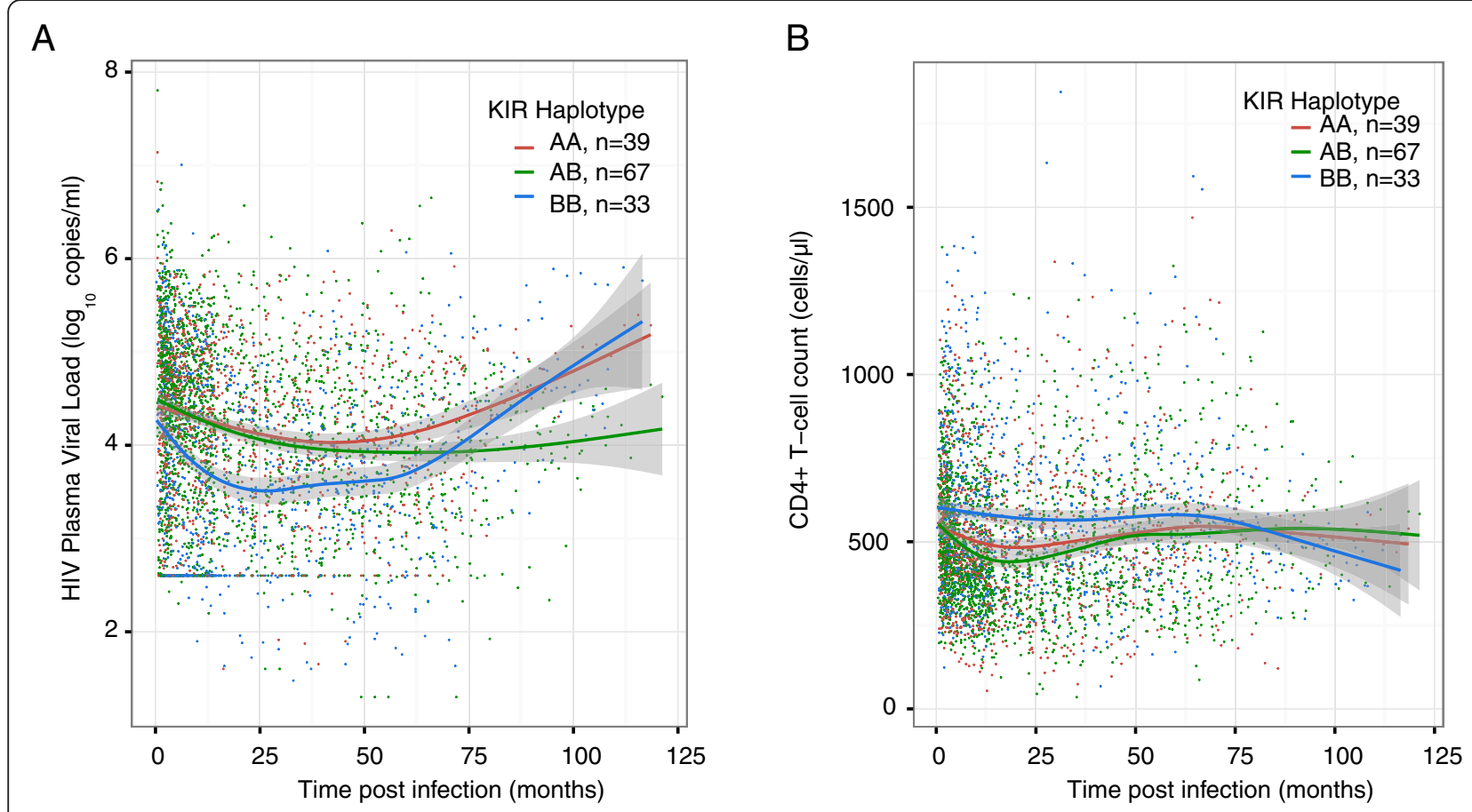

C

D


Fig. 2 KIR haplotype BB is associated with a lower VL and $\mathbf{b}$ higher CD4+ T-cell counts in primary HIV infection. $\mathbf{c}$ The linkage-disequilibrium between KIR genes. $\mathbf{d}$ KIR2DL2 is associated with lower viral loads whilst KIR2DL3 (homozygous or heterozygous) is associated with relatively higher viral loads. Each plot shows the individual viral load or CD4 count measure as well as a LOWESS -smoothed curve fitted to the data as well as the $95 \%$ confidence interval for the curve (grey shading adjacent to coloured lines)

T-cell counts sustained over more than 5 years in a cohort of more than 130 prospectively followed South African women from a homogenous ethnic background.
Lack of association between KIR haplotype and HIV1 acquisition observed here are in contrast to previous smaller studies linking higher activating:inhibitory KIR 
receptor repertoires, and the presence of KIR3DS1 in particular, and genotype $\mathrm{AB}$, with reduced HIV acquisition [10]. The KIR3DS1 gene is infrequent in the population under study, which is typical for populations of African descent [35]. Our study, despite being larger than previous studies, was powered to identify covariates with unadjusted odds ratio $<0.63$ or $>1.6$, hence we may commit type 2 error if true effect sizes exist and are smaller. Although not feasible here, a more robust strategy would have been a prospective cohort analysis.

The association between KIR and HIV disease course is in agreement with immunogenetic [5] and functional studies [36] as well as recent evidence that NK cell function is a feature of HIV-1 control in patients with poor CD8+ T-cell responses [37]. Similarly, in HCV and HTLV1 infection KIR2DL2 modifies HLA mediated effects on disease and is thereby implicated in protective responses [20]. However, our data are in contrast with observations reported by Khakoo et al. on Hepatitis C infection, where KIR2DL3 homozygous individuals had superior resolution [38]. These different outcomes may be explained by differences in pathogenesis between HCV and HIV-1. Our findings are also in contrast to a previous report of lower CD4+ T-cell counts in HIV infected KIR haplotype B carriers [39]. However, this apparent discrepancy may be due to cross-sectional sampling in Jennes et al. [39] leading to a frailty bias in selection of participants and consequent reversal of direction of effect as was recently observed in a separate study [33]. Gaudieri et al. also reported that carriage of either of the haplotype B genes KIR2DS2 or KIR2DL2 was associated with more rapid CD4+ T-cell decline, but they did not specifically assess the combined haplotype [40]. Nevertheless, these findings suggest the potential for population heterogeneity in effect and highlight the challenges in confidently delineating which KIR gene contributes to the haplotype effect.

Several underlying mechanisms may be involved in KIR2DL2 mediated enhancement of HIV control, or reciprocally of KIR2DL3 impairment. Firstly, the presence of KIR2DL2 associated footprints in virus sequenced from KIR2DL2+ donors supports a model in which KIR2DL2 may bind HIV-derived viral peptides presented by HLA-C [17]. In vitro studies using viral peptide variants suggest that selected viral peptides enhance KIR2DL2 binding, resulting in NK cell inhibition and diminished degranulation, hence affording the virus a selection advantage [41]. This may explain why the beneficial effect of KIR2DL2 may be lost later in infection. Whilst differing by only a few amino acids, KIR2DL2 has been reported to have higher affinity for HLA-CI than KIR2DL3, and KIR2DL2/ 2DL3 differ in their sensitivity to peptide bound in the HLA C groove offering a further potential explanation for how subtle differences may explain the divergent effects of KIR2DL2/L3 [42]. Although Korner et al. [15], show that KIR2DL2+ NK cells are functionally more potent in the presence of HLA-C1/C1, because KIR2DL2 mediates inhibitory signals following binding to HLA-C molecules of both HLA-C1 or HLA-C2, the absence of an interaction between KIR2DL2 and HLA-C1/C2 group in our study is compatible with this model [43]. The linkage disequilibrium that we found between KIR2DL2 and KIR2DS2 was consistent with previous studies [44] and implies that a role for KIR2DS2 cannot be excluded. An alternative mechanism is suggested by Schonberg et al. [43] who report that due to differences in timing of KIR2DL2 and KIR2DL1 expression, the presence of KIR2DL2 may affect ligand-instructed NK cell education during development. Finally, KIR2DL2 may act indirectly to alter T-cell recognition of virally infected cells as described in HTLV-1 and HCV infection [20].

Blockade of inhibitory KIR interaction with HLA-C has been pursued as therapeutic strategy in malignancy and viral infection [45]. An antibody that blocks interaction of KIR2DL1/L2/L3/S1/S2 (1-7 F9) with HLA-C, was shown to enhance degranulation of NK cells from HIV infected donors cocultured with target cells in vitro [46]. The enhancement in NK cell degranulation observed with 1-7 F9 was higher amongst NK cells from donors with KIR haplotype B in congruence with our observations. However, precise delineation of the role of KIR2DL2, KIR2DL3 and KIR2DS2 is difficult. This approach has been further developed in acute myeloid leukaemia (AML), multiple myeloma (MM) and lymphoma models [47-49]. A humanised version (lirilumab) was shown to be safe [50] in humans and is currently in clinical trials for treatment of AML (NCT01687387), MM (NCT02252263, NCT01592370), lymphoma (NCT01592 370 ) and some solid organ tumours (NCT01750580, NC T01714739). We speculate that similar approaches may be beneficial in HIV.

In spite of having a pre-determined data analysis plan and consistent viral load and CD4+ T-cell measures across both sub-cohorts, we cannot exclude that this finding is a false positive due to multiple comparisons. Larger studies, that limit analyses to replication of this finding, are required. In addition, this study has other noteworthy limitations. Firstly, it is limited by the lack of resolution of specific KIR alleles and copy number. Secondly, in the case control analysis we were unable to account for the transmitting partners KIR status constraining our ability to confirm previous reports of KIR2DS4*001 carriage in the transmitting partner being linked to enhanced transmission risk [51]. Thirdly, the limited sample size prohibited subgroup analyses, for example, of HLA C1/C2 ligand interactions. Finally, we were unable to link these clinical observations with previously reported flow-cytometry based measures of NK cell function [23] due to the absence of KIR2DL2/L3 specific antibodies used in our previous work. 


\section{Conclusions}

Our data suggest that KIR2DL2 on the B KIR haplotype may mediate measurable control of HIV viraemia and underlies a protective effect through the early years of chronic HIV infection. Larger studies are required to confirm this finding and establish its generalizability. These data also support exploration of using KIR-blocking approaches to manipulate NK cell function in HIV infection.

\section{Additional files}

\section{Additional file 1: Statistical Analysis plan: KIR/HLA and viral control in seroconverters in CAPRISA 004 and CAPRISA050/051/ HEPS. (DOCX $23 \mathrm{~kb}$ ) \\ Additional file 2: Figure S1. KIR haplotype BB is associated with lower viral loads in both subsets of the cohort as shown in upper (CAPRISA 050/051 and CAPRIS002/HEPS) and lower (CAPRISA004) panels respectively. Upper and lower panel shows individual viral load measures (dots) and LOWESS smoothed curve coloured according to donor KIR haplotype with $95 \% \mathrm{Cl}$ shown in grey shading. (PDF $1119 \mathrm{~kb}$ )}

Additional file 3: Figure S2. Cross-sectional viral load measures according to KIR haplotype. Boxes denote interquartile range (box margin) and median (solid line), and whiskers denote range. (PDF $1210 \mathrm{~kb}$ )

Additional file 4: Figure S3. Viral load measures according to HLA-types. . Each panel shows individual viral load or CD4+ T-cell count measures (dots) and LOWESS smoothed curve coloured according to HLA classification as protective, harmful or reference according to previous studies, with $95 \% \mathrm{Cl}$ shown in grey shading. (PDF $1148 \mathrm{~kb}$ )

Additional file 5: Supplementary Table 1. Distribution of KIR2DL2/ KIR2DL3 allele status according to HLA-C ligand groupings. (DOCX $38.3 \mathrm{~kb}$ )

Additional file 6: Figure S4. Viral load $(A-C)$ and $C D 4+T$-cell count (D-F) measures in KIR2DL2/KIR2DL2 (A, D), KIR2DL2/KIR2DL3 (B, E), KIR2DL3/KIR2DL3 (C, F) according to HLA-C1/HLA-C2 ligand grouping. Each panel shows individual viral load or CD4+ T-cell count measures (dots) and LOWESS smoothed curve coloured according to donor HLA C1/C2 allele groupings. (PDF $1822 \mathrm{~kb}$ )

\section{Competing interests}

The authors have no relevant conflict of interests to report.

\section{Authors' contributions}

SSAK, QAK, AK, NG, SS and KM were involved in cohort accrual and clinical follow up. VN, DdAR, HH, CG, and RM performed the KIR and HLA typing. VN, LW, DC, MA, AVSH, WHC, SSAK, and TN led and performed analyses and all authors read and commented on the manuscript. All authors read and approved the final manuscript.

\section{Acknowledgements}

We thank the participants of the CAPRISA study cohorts; women who are dedicated and committed to improving their and their peers' health and who donate samples to make this research possible. We thank Mary Carrington for helpful advice in preparation of this manuscript. This work was supported by the South African HIV/AIDS Research Platform (SHARP), and US National Institutes for Health FIC K01-TW007793. The parent trial (CAPRISA004) was supported by the United States Agency for International Development (USAID), Family Health International (FHI) co-operative agreement \# GPO-A-00-05-00022-00, contract \# 132119, and LIFElab, a biotechnology centre of the South African Department of Science \& Technology. These studies were also supported by the TRAPS (Tenofovir gel Research for AIDS Prevention Science) Program, which is funded by CONRAD co-operative grant \# GP00-0800005-00, subproject agreement \# PPA-09-046. We thank the US National Institutes for Health's Comprehensive International Program of Research on AIDS (CIPRA grant \# Al51794) for the research infrastructure. V.N. was supported by LIFElab, the Columbia University-South Africa Fogarty AIDS International
Training and Research Program (AITRP \#D43 TW000231) and the Rhodes Trust. M.A. is a Distinguished Clinical Scientist of the Doris Duke Charitable Foundation. W.H.C was supported by a Massachusetts General Hospital Physician Scientist Development Award. T.N. holds the South African Research Chair in Systems Biology of HIV/AIDS supported by the South African Department of Science and Technology through the National Research Foundation. T.N. received additional funding from the Victor Daitz Foundation and is a Howard Hughes Medical Institute International Early Career Scientist. VN and AVSH were partially supported through a grant from the Wellcome Trust which supports core facilities (090532/Z/09/Z).

\section{Author details}

${ }^{1}$ Centre for the AIDS Programme of Research in South Africa (CAPRISA), University of KwaZulu-Natal, Durban, South Africa. ${ }^{2}$ Wellcome Trust Centre for Human Genetics, Nuffield Department of Medicine, University of Oxford, Oxford, UK. ${ }^{3} \mathrm{HIV}$ Pathogenesis Programme, University of KwaZulu-Natal, Durban, South Africa. ${ }^{4}$ National Institute of Communicable Diseases, Sandringham, South Africa. ${ }^{5}$ University of the Witwatersrand, Johannesburg, South Africa. ${ }^{6}$ University of Cape Town, Cape Town, South Africa. ${ }^{7}$ City University of New York - Medgar Evers College, New York, USA. ${ }^{8}$ Ragon Institute of MGH, MIT and Harvard University, Boston, USA. ${ }^{9}$ Mailman School of Public Health, Columbia University, New York, USA. ${ }^{10}$ Leibniz Institute for Experimental Virology, Heinrich Pette Institute, Hamburg, Germany. ${ }^{11}$ KwaZulu-Natal Research Institute for Tuberculosis and HIV, University of KwaZulu-Natal, Durban, South Africa. ${ }^{12}$ Max Planck Institute for Infection Biology, Chariteplatz, D-10117 Berlin, Germany. ${ }^{13}$ Division of Virology, School of Pathology, Faculty of Health Sciences, University of the Witwatersrand, Johannesburg, South Africa.

Received: 13 August 2015 Accepted: 18 January 2016 BP?

\section{References}

1. Miyazawa M, Lopalco L, Mazzotta F, Lo Caputo S, Veas F, Clerici M. The 'immunologic advantage' of HIV-exposed seronegative individuals. Aids. 2009;23(2):161-75.

2. Shea PR, Shianna KV, Carrington M, Goldstein DB. Host genetics of HIV acquisition and viral control. Annu Rev Med. 2013;64:203-17.

3. Walker BD, Yu XG. Unravelling the mechanisms of durable control of HIV-1. Nat Rev Immunol. 2013;13(7):487-98.

4. Apps R, Qi Y, Carlson JM, Chen H, Gao X, Thomas R, et al. Influence of HLA-C expression level on HIV control. Science. 2013;340(6128):87-91.

5. Martin MP, Gao X, Lee JH, Nelson GW, Detels R, Goedert JJ, et al. Epistatic interaction between KIR3DS1 and HLA-B delays the progression to AIDS. Nat Genet. 2002:31(4):429-34.

6. Fellay J, Shianna KV, Ge D, Colombo S, Ledergerber B, Weale M, et al. A whole-genome association study of major determinants for host control of HIV-1. Science. 2007;317(5840):944-7.

7. Rajalingam R. Overview of the killer cell immunoglobulin-like receptor system. Methods Mol Biol. 2012;882:391-414.

8. Klein J, Sato A. The HLA system. First of two parts. N Engl J Med. 2000; 343(10):702-9.

9. Boulet S, Sharafi S, Simic N, Bruneau J, Routy JP, Tsoukas CM, et al. Increased proportion of KIR3DS1 homozygotes in HIV-exposed uninfected individuals. Aids. 2008;22(5):595-9.

10. Jennes W, Verheyden S, Demanet C, Adje-Toure CA, Vuylsteke B, Nkengasong JN, et al. Cutting edge: resistance to HIV-1 infection among African female sex workers is associated with inhibitory KIR in the absence of their HLA ligands. J Immunol. 2006;177(10):6588-92.

11. Paximadis M, Minevich G, Winchester R, Schramm DB, Gray GE, Sherman GG, et al. KIR-HLA and maternal-infant HIV-1 transmission in sub-Saharan Africa. PLoS One. 2011;6(2):e16541.

12. Ravet $\mathrm{S}, \mathrm{Scott}-$ Algara $\mathrm{D}, \mathrm{Bonnet} \mathrm{E}$, Tran HK, Tran T, Nguyen N, et al. Distinctive NK-cell receptor repertoires sustain high-level constitutive NK-cell activation in HIV-exposed uninfected individuals. Blood. 2007;109(10):4296-305.

13. Scott-Algara D, Truong LX, Versmisse P, David A, Luong TT, Nguyen NV, et al. Cutting edge: increased NK cell activity in HIV-1-exposed but uninfected Vietnamese intravascular drug users. J Immunol. 2003;171(11):5663-7.

14. Alter $G$, Teigen N, Ahern R, Streeck H, Meier A, Rosenberg ES, et al. Evolution of innate and adaptive effector cell functions during acute HIV-1 infection. J Infect Dis. 2007;195(10):1452-60. 
15. Korner C, Granoff ME, Amero MA, Sirignano MN, Vaidya SA, Jost S, et al. Increased frequency and function of KIR2DL1-3 NK cells in primary HIV-1 infection are determined by HLA-C group haplotypes. Eur J Immunol. 2014; 44(10):2938-48. doi:10.1002/eji.201444751.

16. Thomas R, Apps R, Qi Y, Gao X, Male V, O'HUigin C, et al. HLA-C cell surface expression and control of HIV/AIDS correlate with a variant upstream of HLA-C. Nat Genet. 2009;41(12):1290-4.

17. Alter $G$, Heckerman D, Schneidewind A, Fadda L, Kadie CM, Carlson JM, et al. HIV-1 adaptation to NK-cell-mediated immune pressure. Nature. 2011; 476(7358):96-100.

18. Poon K, Montamat-Sicotte D, Cumberbatch N, McMichael AJ, Callan MF. Expression of leukocyte immunoglobulin-like receptors and natural killer receptors on virus-specific CD8+ T cells during the evolution of Epstein-Barr virus-specific immune responses in vivo. Viral Immunol. 2005;18(3):513-22.

19. van der Veken LT, Diez Campelo M, van der Hoorn MA, Hagedoorn RS, van Egmond HM, van Bergen J, et al. Functional analysis of killer lg-like receptor-expressing cytomegalovirus-specific CD8+ T cells. J Immunol. 2009; 182(1):92-101.

20. Seich Al Basatena NK, Macnamara A, Vine AM, Thio CL, Astemborski J, Usuku K, et al. KIR2DL2 enhances protective and detrimental HLA class I-mediated immunity in chronic viral infection. PLoS pathogens. 2011;7(10):e1002270.

21. Alter G, Rihn S, Streeck H, Teigen N, Piechocka-Trocha A, Moss K, et al. Ligand-independent exhaustion of killer immunoglobulin-like receptor-positive CD8+ T cells in human immunodeficiency virus type 1 infection. J Virol. 2008; 82(19):9668-77.

22. Naranbhai V, Abdool Karim SS, Altfeld M, Samsunder N, Durgiah R, Sibeko S, et al. Innate Immune Activation Enhances HIV Acquisition in Women, Diminishing the Effectiveness of Tenofovir Microbicide Gel. J Infect Dis. 2012;206(7):993-1001.

23. Naranbhai V, Altfeld M, Abdool Karim Q, Ndung'u T, Abdool Karim SS, Carr WH. Natural killer cell function in women at high risk for HIV acquisition: insights from a microbicide trial. Aids. 2012;26(14):1745-53.

24. Naranbhai V, Altfeld M, Karim SS, Ndung'u T, Karim QA, Carr WH. Changes in Natural Killer cell activation and function during primary HIV-1 Infection. PLoS One. 2013;8(1):e53251.

25. Garrett NJ, Werner L, Naicker N, Naranbhai V, Sibeko S, Samsunder N, et al. HIV Disease Progression in Seroconvertors from the CAPRISA 004 Tenofovir Gel Pre-exposure Prophylaxis Trial. J Acquir Immune Defic Syndr. 2015;68(1):55-61.

26. Mlisana K, Werner L, Garrett NJ, McKinnon LR, van Loggerenberg F, Passmore JA, et al. Rapid Disease Progression in HIV-1 Subtype C-Infected South African Women. Clin Infect Dis. 2014;59(9):1322-31. doi:10.1093/cid/ciu573.

27. Abdool Karim Q, Abdool Karim SS, Frohlich JA, Grobler AC, Baxter C, Mansoor LE, et al. Effectiveness and safety of tenofovir gel, an antiretroviral microbicide, for the prevention of HIV infection in women. Science. 2010; 329(5996):1168-74

28. Abdool Karim Q, Kharsany AB, Frohlich JA, Werner L, Mlotshwa M, Madlala BT, et al. HIV incidence in young girls in KwaZulu-Natal, South Africa-public health imperative for their inclusion in HIV biomedical intervention trials. AIDS Behav. 2012;16(7):1870-6.

29. van Loggerenberg F, Mlisana K, Williamson C, Auld SC, Morris L, Gray CM, et al. Establishing a cohort at high risk of HIV infection in South Africa: challenges and experiences of the CAPRISA 002 acute infection study. PLoS One. 2008;3(4):e1954

30. Naicker N, Kharsany AB, Werner L, van Loggerenberg F, Mlisana K, Garrett N, et al. Risk factors for HIV acquisition in high risk women in a generalised epidemic setting. AIDS Behav. 2015;19(7):1305-16. doi:10.1007/s10461-015-1002-5.

31. Abdool Karim Q, Abdool Karim SS, Frohlich JA, Grobler AC, Baxter C, Mansoor LE, et al. Effectiveness and safety of tenofovir gel, an antiretroviral microbicide, for the prevention of HIV infection in women. Science. 2010; 329(5996):1168-74

32. Hong HA, Loubser AS, de Assis RD, Naranbhai V, Carr W, Paximadis M, et al. Killer-cell immunoglobulin-like receptor genotyping and HLA killer-cell immunoglobulin-like receptor-ligand identification by real-time polymerase chain reaction. Tissue Antigens. 2011;78(3):185-94.

33. McLaren PJ, Coulonges C, Ripke S, van den Berg L, Buchbinder S, Carrington M, et al. Association study of common genetic variants and HIV-1 acquisition in 6,300 infected cases and 7,200 controls. PLoS Pathog. 2013;9(7):e1003515.

34. Lane J, McLaren PJ, Dorrell L, Shianna KV, Stemke A, Pelak K, et al. A genome-wide association study of resistance to HIV infection in highly exposed uninfected individuals with hemophilia A. Hum Mol Genet. 2013; 22(9):1903-10
35. Norman PJ, Stephens HA, Verity DH, Chandanayingyong D, Vaughan RW. Distribution of natural killer cell immunoglobulin-like receptor sequences in three ethnic groups. Immunogenetics. 2001;52(3-4):195-205.

36. Alter G, Martin MP, Teigen N, Carr WH, Suscovich TJ, Schneidewind A, et al. Differential natural killer cell-mediated inhibition of HIV-1 replication based on distinct KIR/HLA subtypes. J Exp Med. 2007;204(12):3027-36.

37. Scott-Algara D, Didier C, Arnold V, Cummings JS, Sáez-Cirión A, Boufassa F, et al. Post-Treatment Controllers Have Particular NK Cells With High Anti-HIV Capacity: VISCONTI Study. In: CROI 2015. Seattle, Washington; 2015

38. Knapp S, Warshow U, Hegazy D, Brackenbury L, Guha IN, Fowell A, et al. Consistent beneficial effects of killer cell immunoglobulin-like receptor 2DL3 and group 1 human leukocyte antigen-C following exposure to hepatitis $C$ virus. Hepatology. 2010;51(4):1168-75.

39. Jennes W, Verheyden S, Demanet C, Menten J, Vuylsteke B, Nkengasong JN, et al. Low CD4+ T cell counts among African HIV-1 infected subjects with group B KIR haplotypes in the absence of specific inhibitory KIR ligands. PLoS One. 2011;6(2):e17043.

40. Gaudieri S, Nolan D, McKinnon E, Witt CS, Mallal S, Christiansen FT. Associations between KIR epitope combinations expressed by $\mathrm{HLA}-\mathrm{B} /-\mathrm{C}$ haplotypes found in an HIV-1 infected study population may influence NK mediated immune responses. Mol Immunol. 2005;42(4):557-60.

41. Fadda L, Korner C, Kumar S, van Teijlingen NH, Piechocka-Trocha A, Carrington M, et al. HLA-CW*0102-restricted HIV-1 p24 epitope variants can modulate the binding of the inhibitory KIR2DL2 receptor and primary NK cell function. PLoS Pathog. 2012;8(7):e1002805.

42. Cassidy S, Mukherjee S, Myint TM, Mbiribindi B, North $H$, Traherne J, et al. Peptide selectivity discriminates NK cells from KIR2DL2- and KIR2DL3-positive individuals. Eur J Immunol. 2015;45(2):492-500.

43. Schonberg K, Sribar M, Enczmann J, Fischer JC, Uhrberg M. Analyses of HLA-C-specific KIR repertoires in donors with group A and B haplotypes suggest a ligand-instructed model of NK cell receptor acquisition. Blood. 2011;117(1):98-107.

44. Moesta AK, Parham P. Diverse functionality among human NK cell receptors for the C1 epitope of HLA-C: KIR2DS2, KIR2DL2, and KIR2DL3. Frontiers in immunology. 2012;3:336

45. Sola C, Andre P, Lemmers C, Fuseri N, Bonnafous C, Blery M, et al. Genetic and antibody-mediated reprogramming of natural killer cell missing-self recognition in vivo. Proc Natl Acad Sci U S A. 2009;106(31):12879-84.

46. Johansson SE, Hejdeman B, Hinkula J, Johansson MH, Romagne F, Wahren B, et al. NK cell activation by KIR-binding antibody 1-7 F9 and response to HIV-infected autologous cells in viremic and controller HIV-infected patients. Clin Immunol. 2010;134(2):158-68.

47. Benson Jr DM, Bakan CE, Zhang S, Collins SM, Liang J, Srivastava S, et al. IPH2101, a novel anti-inhibitory KIR antibody, and lenalidomide combine to enhance the natural killer cell versus multiple myeloma effect. Blood. 2011; 118(24):6387-91.

48. Romagne F, Andre P, Spee P, Zahn S, Anfossi N, Gauthier L, et al. Preclinical characterization of 1-7 F9, a novel human anti-KIR receptor therapeutic antibody that augments natural killer-mediated killing of tumor cells. Blood. 2009;114(13):2667-77.

49. Kohrt HE, Thielens A, Marabelle A, Sagiv-Barfi I, Sola C, Chanuc F, et al. Anti-KIR antibody enhancement of anti-lymphoma activity of natural killer cells as monotherapy and in combination with anti-CD20 antibodies. Blood. 2014; 123(5):678-86.

50. Vey N, Bourhis JH, Boissel N, Bordessoule D, Prebet T, Charbonnier A, et al. A phase 1 trial of the anti-inhibitory KIR mAb IPH2101 for AML in complete remission. Blood. 2012;120(22):4317-23.

51. Merino A, Malhotra R, Morton M, Mulenga J, Allen S, Hunter E, et al. Impact of a functional KIR2DS4 allele on heterosexual HIV-1 transmission among discordant Zambian couples. J Infect Dis. 2011;203(4):487-95. 